\title{
Características personales, clínicas y sociales en pacientes con estancia hospitalaria prolongada en los pabellones de pacientes agudos del Hospital Víctor Larco Herrera
}

Roger Abanto1,a, Glauco Valdivieso-Jiménez ${ }^{1, b}$, Adrián Arévalo $^{1,2, b}$, Sandra Orihuela ${ }^{1,3, b}$

RESUMEN

Objetivo: Identificar las características generales que influyen en la estancia hospitalaria prolongada en los pabellones de pacientes agudos y su comparación sobre el motivo de ingreso en una institución de Salud Mental. Materiales y métodos: Estudio descriptivo, retrospectivo, y transversal, realizado en el Hospital Víctor Larco Herrera. Con una población entre 18 a 65 años con una estancia mayor a 60 días en los pabellones de pacientes agudos. Se realizó revisión de historias clínicas, recolección de datos en ficha estructurada y análisis estadístico mediante SPSS 22.0. Principales medidas de resultados: frecuencias, media y valor de p mediante chi cuadrado en las variables características personales, clínicas y sociales.

Resultados: Fueron 52 \% de pacientes internados que cumplían estancia mayor a 60 días, cuyas edades fueron: jóvenes y jóvenes adultos $45 \%$, mediana 35,89, DE=10,45. Mujeres, un $60 \%$; instrucción secundaria, $61 \%$; solteros, 81 \%; desocupación laboral, 74 \%. Ingresados por Emergencia, 74 \%, reingresos, 55 \%, de Lima, 53 \%. El diagnóstico más frecuente fue esquizofrenia en $62 \%$; comorbilidad, 61 \%; presentación con agresividad, $64 \%$; evolución estacionaria, $63 \%$; ingreso por motivo judicial,47 \% y clínico en $47 \%$. El tratamiento brindado a los pacientes fue principalmente de combinación de fármacos en 74,2 \%. Sin abandono sociofamiliar, el 52 \%; acompañados por familiares, 66 \%; acompañados por policía, 71,4 \%; familia disfuncional, 72,6 \%; soporte por padres, 51,6 \% y pertenencia al SIS el $92 \%$. Existe mayor significancia estadística en el sexo, estado civil, procedencia, vía de ingreso, tipo o condición de paciente (nuevo o reingreso), acompañamiento de familiares y de otras personas al ingreso.

Conclusiones: La estancia hospitalaria está afectada por factores personales como el sexo y estado civil en pacientes judicializados. La procedencia, factores clínicos como la vía y la condición de ingreso; y también sociales como el acompañamiento de familiar y de otros no emparentados, influyen en pacientes ingresados por motivo clínico.

Palabras clave: Pacientes mentales; hospitalización; salud mental (Fuente: DeCS BIREME).

\section{Personal, clinical and social characteristics of inpatients with long hospital stay in the acute wards of the Hospital Víctor Larco Herrera}

\section{ABSTRACT}

Objective: To identify the general characteristics that influence a long hospital stay in the acute wards of a mental health institution and to compare them with the reason for admission.

Materials and methods: A descriptive, retrospective and cross-sectional study was conducted at the Hospital Víctor Larco Herrera in patients between 18 and 65 years of age staying longer than 60 days in the acute wards. Medical records review, data collection in structured files, and statistical analysis through IBM SPSS Statistics Base 22.0 were performed. Main outcome measures were the frequencies, mean and $p$ value (obtained by chi square test) in the variables of personal, clinical and social characteristics.

1. Hospital Victor Larco Herrera. Lima, Perú

2. Universidad Nacional Mayor de San Marcos, Facultad de Medicina "San Fernando". Lima, Perú.

3. Universidad Ricardo Palma, Facultad de Medicina. Lima, Perú.

a. Médico Psiquiatra, Jefe del Departamento de Epidemiología.

b. Médico Residente de Psiquiatría. 
Results: Out of the total number of inpatients, $52 \%$ were staying longer than 60 days, $45 \%$ were youths and young adults (median $=35.89, \mathrm{SD}=10.45$ ), $60 \%$ were women, $61 \%$ had secondary education, $81 \%$ were single, $74 \%$ were unemployed, $74 \%$ were admitted through the emergency department, $55 \%$ were readmitted, $53 \%$ were from Lima, $62 \%$ had schizophrenia which was the most frequent diagnosis, $61 \%$ showed comorbidities, $64 \%$ presented an aggressive behavior on admission, $63 \%$ showed a stable progression, $47 \%$ were admitted due to legal reasons, $47 \%$ were admitted due to clinical reasons, $74.2 \%$ mainly received combination therapies, $52 \%$ did not experience social or family abandonment, $66 \%$ were accompanied by their relatives, $71.4 \%$ were accompanied by the police, $72.6 \%$ had a dysfunctional family, $51.6 \%$ were supported by their parents, and $92 \%$ were covered by the Comprehensive Health Service (SIS). There is greater statistical significance in gender, marital status, origin, admission route, patient type or condition (new or readmitted), and company of relatives and other persons at admission.

Conclusions: Hospital stay is affected by personal factors such as gender and marital status in patients admitted due to legal reasons. The origin, clinical factors such as the admission route and condition, and social factors such as the company of relatives and other persons, have influence on patients admitted due to clinical reasons.

Keywords: Mental patients; hospitalization; mental health (Source: MeSH NLM). 


\section{INTRODUCCIÓN}

Con el advenimiento de la atención administrada y los principios de desinstitucionalización, se ha concedido gran importancia en el tratamiento limitado en el tiempo, tanto en hospitales como en centros ambulatorios psiquiátricos. Los medicamentos psicotrópicos más eficaces y un enfoque en la estabilización de los síntomas durante la hospitalización han dado como resultado una disminución diaria del censo y una reducción en la duración media de la estancia en muchas instituciones psiquiátricas ${ }^{(1,2)}$. Sin embargo, un subconjunto de pacientes continúa utilizando recursos de salud mental en un grado desproporcionado, medido por la duración de la hospitalización o las admisiones repetidas con el tiempo. Este uso desproporcionado es problemático por dos razones. Primero, las estancias hospitalarias más largas no significan necesariamente una mejor atención de la salud mental, ajuste social mejorado, psicopatología disminuida ni menos readmisiones. Esto plantea la cuestión de por qué una cohorte de pacientes sigue requiriendo hospitalización prolongada, así como la razón de ser de la extensión de los servicios cuando la utilidad de hacerlo es cuestionable. En segundo lugar, dado que los pacientes con estancias hospitalarias más largas también suelen tener más admisiones y estancias más prolongadas, se han planteado preguntas sobre si estos pacientes están recibiendo servicios integrales de salud mental proporcionados de manera competente ${ }^{(2,3)}$.

En nuestro país, según el Informe Defensorial $N^{\circ} 102$ (2005), existe una problemática en relación al número de pacientes judicializados en la mayoría de las instituciones de salud mental, cuyo internamiento genera limitaciones en el sistema hospitalario. Dentro de estas instituciones se encuentra el Hospital Víctor Larco Herrera donde se ha reportado a 16 pacientes internados con esta condición; el Hospital Hermilio Valdizán con 6; el Instituto Nacional de Salud Mental con 1 , entre otros. A la actualidad ha variado el número de los mismos ${ }^{(4,5)}$.

La falta de vivienda, el apoyo de la comunidad, la severidad de estresores psicosociales o el desempleo para los pacientes que requieren atención a largo plazo se citan comúnmente entre las razones que impiden las altas hospitalarias y amplían las estancias hospitalarias en unidades de psiquiatría aguda. Como resultado, una proporción importante de camas de estos servicios se ocupan inapropiadamente debido a la ausencia de ajustes alternativos adecuados. Así también, muchas veces estos pacientes sufren diversas comorbilidades clínicas no mentales que contribuyen al impacto de la estancia prolongada ${ }^{(6,7)}$.

En relación a algunos trabajos realizados como antecedentes resaltamos el de Afilalo et al. (8) quienes concluyeron que la mayoría de los pacientes en internamiento prolongado no contaban con soporte social y entre los que requerían atención aguda e internamiento prolongado los problemas psiquiátricos más frecuentes fueron delirios o alucinaciones (34\%), incapacidad de tomar medicamentos de forma independiente $(23,6 \%)$ y control inadecuado de la agresión o impulsividad $(16,5 \%)$. Adegunloye et al. ${ }^{\left({ }^{9}\right)}$ estudiaron que alrededor del $51,8 \%$ de las admisiones totales fueron mujeres, el 68,5\% pertenecía al grupo de 20 a 40 años, el $58 \%$ eran solteros y el $36,1 \%$ eran estudiantes. El diagnóstico más frecuente fue esquizofrenia en $55 \%$, y $58 \%$ de los pacientes presentaron primer episodio de enfermedad psiquiátrica.

La duración de la estancia se correlacionó significativamente con el estado civil y el primer episodio de enfermedad psiquiátrica. Un buen apoyo social puede acortar la duración de la estancia en la admisión psiquiátrica, por lo tanto, el suministro de buenas estrategias de psicoeducación para todos los pacientes y sus familias, así como la prevención eficaz de la recaída para el primer episodio de pacientes agudos fueron recomendables. Zhang et al. (10) concluyeron que las manifestaciones conductuales de la enfermedad y la falta de estructuras de apoyo social predijeron la prolongación de la estancia. Herr et al. (11) mencionaron que el tratamiento con terapia electroconvulsiva, consultas médicas, subempleo, demencia, disposición a un lugar distinto del hogar, ausencia de alcohol o abuso de drogas y presencia de psicosis sin síntomas afectivos resultaron predictores de hospitalización prolongada. Addisu et al. (12) resaltaron que los diagnósticos más frecuentes fueron esquizofrenia y otros trastornos psicóticos $(27,6 \%)$, y trastorno bipolar $(23,4 \%)$ los que condicionarían el internamiento a un tiempo mayor del esperado.

El objetivo de este trabajo fue el analizar e identificar las características asociadas a la estancia hospitalaria prolongada en los pabellones 01 (varones) y 20 (mujeres) del Hospital Víctor Larco Herrera considerando las variables como características personales, clínicas y sociales; así mismo, identificar la significancia según el motivo de ingreso que condujo 
al internamiento prolongado. Debido a la falta de estudios relacionados a salud mental en un hospital psiquiátrico, los autores consideramos importante el estudio de estas características que perjudican la efectividad y atención de pacientes que realmente requieren una hospitalización oportuna.

\section{MATERIALES Y MÉTODOS}

Este estudio fue descriptivo, retrospectivo y transversal. La unidad de análisis fueron las historias clínicas de los pacientes de entre 18 a 65 años de edad con una estancia hospitalaria de más de 60 días en los pabellones uno (varones) y veinte (mujeres) del Hospital Víctor Larco Herrera considerando lo establecido por la Ley General de Salud Mental. Se revisaron las historias clínicas para conocer los datos relacionados a las variables a investigar: características personales (edad, sexo, grado de instrucción, ocupación y procedencia), clínicos (vía de ingreso, tipo de paciente, diagnóstico, signos y síntomas, evolución, tratamiento y motivo de ingreso), sociales (con o sin familia, tipo de familia y nivel socioeconómico). Para tal fin se desarrolló un instrumento de recolección de los datos. Los criterios de inclusión fueron todos los pacientes hospitalizados en los pabellones uno y veinte del Hospital Víctor Larco Herrera que al momento de la recolección de la información tenían una estancia mayor a 60 días.

Los criterios de exclusión fueron aquellos pacientes con comorbilidad médica general y que se encontraban en el momento del estudio en UCE (Unidad de Cuidados Especiales), los pacientes albergados (pabellones de crónicos), del Servicio de Adicciones y los de Emergencia. Se solicitó la autorización de la Oficina de Docencia del hospital y aprobación del Comité de Ética para ejecutar el procedimiento. Se tuvo como fuente de información las historias clínicas reuniendo los datos durante el mes de diciembre de 2015. La primera etapa constituyó de una prueba piloto para afinar el instrumento y generar observaciones. En la segunda etapa correspondió a dar paso a un panel con médicos expertos, a fin de valorar los aportes y comentarios, y dar mayor confiabilidad al instrumento para posteriormente validarlo. Se analizó la base datos en software SPSS 22.0 en el que se tabuló en tablas de frecuencia y se aplicó la prueba estadística chi cuadrado.

\section{RESULTADOS}

Del total de los pacientes hospitalizados en los pabellones 1 y 20 , resultaron ser el $52 \%$ aquellos con un tiempo mayor a 60 días de internamiento.

Se encontró que los grupos etarios más afectados fueron los jóvenes y jóvenes adultos hasta en un $45 \%$, mediana 35,89 y desviación estándar (DE) de 10,45. En relación al sexo, el más afectado fue el sexo femenino, hasta en un $60 \%$. El nivel de instrucción de los pacientes hospitalizados mayormente es el de secundaria, con $61 \%$, seguido el de primaria con un $31 \%$. Respecto al estado civil de los pacientes mayormente son solteros en un $81 \%$, seguido de casados en un $13 \%$. En cuanto a la ocupación o trabajo de los pacientes, se encontró en mayor frecuencia desocupados en un $74 \%$.

Respecto a las características clínicas, se identificó que la vía de ingreso al servicio de hospitalización en dos terceras partes fue por Emergencia en un $74 \%$, y un $26 \%$ por la Consulta Externa. Según el tipo de pacientes que ingresaron, mayormente, son reingresos en un $54,8 \%$. Así mismo, el lugar de procedencia de estos pacientes es de Lima en un 53 \% y procedentes de otras provincias en un $44 \%$. El diagnóstico más frecuente de hospitalización fue esquizofrenia en un $62 \%$, seguido de los trastornos de personalidad $19,4 \%$, trastorno mental orgánico $12,8 \%$, trastorno de sustancias psicoactivas $3,2 \%$ y trastorno bipolar $1,6 \%$. El tipo de diagnóstico fue de comorbilidad en un $61 \%$ a diferencia de un diagnóstico único $37,1 \%$.

Respecto a la forma de presentación (signos y síntomas) más frecuente al ingreso de los pacientes hospitalizados se evidenció mayor frecuencia de agresividad en un 64 \%. En relación a la evolución clínica fue mayormente estacionaria en un $62,9 \%$ y favorable en un $35,5 \%$. Según el motivo de ingreso de los pacientes hospitalizados en ambos pabellones fue por motivo judicial en un $47 \%$ y clínico en un $47 \%$. El tratamiento brindado a los pacientes fue principalmente de combinación de fármacos por vía oral en $74,2 \%$, sólo antipsicóticos atípicos $8,1 \%$, clozapina $8,1 \%$, típicos 3,2 \%, ansiolíticos $4,8 \%$ y otros medicamentos 1,8 \% (Tabla 1). 
Características personales, clínicas y sociales en pacientes con estancia hospitalaria prolongada en los pabellones de pacientes agudos del Hospital Víctor Larco Herrera

Tabla 1. Características clínicas de pacientes con larga estancia hospitalaria en pabellones de pacientes agudos del HVLH

\begin{tabular}{|c|c|c|}
\hline Características clínicas & $\mathbf{N}$ & $\%$ \\
\hline \multicolumn{3}{|l|}{ Vía de ingreso } \\
\hline Consultorio externo & 16 & 25.8 \\
\hline Emergencia & 46 & 74.2 \\
\hline \multicolumn{3}{|l|}{ Tipo de paciente } \\
\hline Nuevo & 28 & 45.2 \\
\hline Reingreso & 34 & 54.8 \\
\hline \multicolumn{3}{|l|}{ Lugar de procedencia } \\
\hline Lima & 33 & 53.2 \\
\hline Provincias & 27 & 43.5 \\
\hline No consignado & 2 & 3.2 \\
\hline \multicolumn{3}{|l|}{ Diagnóstico clínico } \\
\hline Esquizofrenia & 39 & 62.9 \\
\hline Trastorno bipolar & 1 & 1.6 \\
\hline Trastorno de personalidad & 12 & 19.4 \\
\hline Trastorno por SPA & 2 & 3.2 \\
\hline TMO/Retardo mental & 8 & 12.8 \\
\hline \multicolumn{3}{|l|}{ Tipo de diagnóstico } \\
\hline Único & 38 & 61.3 \\
\hline Comórbido & 23 & 37.1 \\
\hline No consignado & 1 & 1.8 \\
\hline \multicolumn{3}{|l|}{ Signos y síntomas al ingreso } \\
\hline Con agresividad & 40 & 64.5 \\
\hline Sin agresividad & 22 & 35.5 \\
\hline \multicolumn{3}{|l|}{ Evolución clínica } \\
\hline Favorable & 22 & 35.5 \\
\hline Desfavorable & 1 & 1.6 \\
\hline Estacionaria & 39 & 62.9 \\
\hline \multicolumn{3}{|l|}{ Motivo de ingreso } \\
\hline No consignado & 1 & 1.6 \\
\hline Clínico & 29 & 46.8 \\
\hline Judicial & 29 & 46.8 \\
\hline Policía/fiscal & 3 & 4.8 \\
\hline \multicolumn{3}{|l|}{ Tratamiento recibido } \\
\hline A. típicos & 2 & 3.2 \\
\hline A. atípicos & 5 & 8.1 \\
\hline Clozapina & 5 & 8.1 \\
\hline Ansiolíticos & 3 & 4.8 \\
\hline Combinación & 46 & 74.2 \\
\hline Otros & 1 & 1.8 \\
\hline
\end{tabular}


Correspondiente a las características sociales, la condición familiar actual de estos pacientes fue sin abandono socio familiar en un $52 \%$ y con abandono socio familiar en un $45 \%$. Respecto a la persona que acompañó al ingreso de estos pacientes fueron con familiar en un $66 \%$ y sin familiar en un $23 \%$. Al considerar a la persona que acompaño al ingreso de los pacientes sin familiar, fue de al menos un policía en un 71,4\%. Relacionado al tipo de familia, el resultado fue de disfuncional en un $72,6 \%$. Para los hallazgos del familiar de soporte de estos, son los padres en un 51,6\%. Finalmente, se muestra que un $92 \%$ de los mismos tienen Seguro Integral de Salud (SIS), además, existe un 6,4 \% de pacientes en condición C y D, es decir, pobres y extremos pobres, pero que aún no contaban con el Seguro Integral de Salud (SIS) (Tabla 2).

Tabla 2. Características sociales de pacientes con larga estancia hospitalaria en pabellones de pacientes agudos del HVLH

\begin{tabular}{lcc} 
Caracteristicas sociales & N & $\%$ \\
\hline Condición familiar actual & & \\
No consignado & 2 & 3.2 \\
Con abandono sociofamiliar & 28 & 45.2 \\
Sin abandono sociofamiliar & 32 & 51.6 \\
\hline Acompañante al ingreso & & \\
No consignado & 7 & 11.3 \\
Con familia & 41 & 66.1 \\
Sin familia & 14 & 22.6 \\
\hline Acompañante al ingreso sin familia & & \\
No consignado & 2 & 14.3 \\
Policía & 10 & 71.4 \\
Amigo & 1 & 7.1 \\
Personal de salud & 1 & 7.1 \\
Tipo de familia & & \\
No consignado & 12 & 19.4 \\
Funcional & 5 & 8.1 \\
Disfuncional & 45 & 72.6 \\
\hline Nivel socioeconómico & & \\
No consignado & 22 & 1.6 \\
C & 1 & 1.6 \\
D & 39 & 4.8 \\
SIS & 62 & 91.9 \\
\hline Familiares con los que cuenta & & \\
No consignado & 81.6 \\
Pareja & 32 & 8.1 \\
Padres & 5 & 1.6 \\
Hermanos & 13 & \\
\hline Hijos & & \\
Otros & & \\
& & \\
\hline
\end{tabular}

En el análisis de las variables correspondientes a las características demográficas comparadas con los motivos de ingreso para estancia prolongada, se encontró que existe mayor significancia estadística en el sexo y estado civil, varones ( $\times 2$ : $9,839 ; \mathrm{p}: 0,020)$ y solteros $(\times 2: 25,256 ; \mathrm{p}: 0,014)$ con la manera de ingreso judicial. Por otro lado, hay significancia estadística en la procedencia, sobre todo en aquellos de Lima (x2: 14,908; p: 0,021) que ingresan por motivo clínico (Tabla 3). 
Características personales, clínicas y sociales en pacientes con estancia hospitalaria prolongada en los pabellones de pacientes agudos del Hospital Víctor Larco Herrera

Tabla 3. Análisis comparativo de características demográficas con el motivo de ingreso en pacientes con internamiento prolongado

\begin{tabular}{|c|c|c|c|c|c|c|c|}
\hline \multicolumn{8}{|c|}{ Motivo de Ingreso } \\
\hline & & $\begin{array}{l}\text { No } \\
\text { Consignado }\end{array}$ & Clínico & Judicial & $\begin{array}{l}\text { Policial, } \\
\text { Fiscal }\end{array}$ & $\mathbf{X} 2$ & $\mathbf{P}$ \\
\hline \multirow[t]{2}{*}{ Sexo } & Masculino & 1 & 14 & 22 & 0 & 9,839 & ${ }^{*} 0,020$ \\
\hline & Femenino & 0 & 15 & 7 & 3 & & \\
\hline Grado de & Analfabeto & 0 & 1 & 0 & 0 & 3,319 & 0,950 \\
\hline \multirow[t]{3}{*}{ instrucción } & Primaria & 0 & 8 & 10 & 1 & & \\
\hline & Secundaria & 1 & 17 & 18 & 2 & & \\
\hline & Superior & 0 & 3 & 1 & 0 & & \\
\hline \multirow[t]{5}{*}{ Estado civil } & No consignado & 0 & 0 & 0 & 1 & 25,256 & ${ }^{*} 0,014$ \\
\hline & Soltero & 1 & 24 & 24 & 1 & & \\
\hline & Casado o conviviente & 0 & 3 & 4 & 1 & & \\
\hline & Divorciado o separado & 0 & 2 & 0 & 0 & & \\
\hline & Viudo & 0 & 0 & 1 & 0 & & \\
\hline \multirow[t]{4}{*}{ Ocupación } & No consignado & 0 & 1 & 1 & 0 & 15,114 & 0,088 \\
\hline & Dependiente & 0 & 0 & 4 & 0 & & \\
\hline & Independiente & 1 & 2 & 7 & 0 & & \\
\hline & Sin ocupación & 0 & 26 & 17 & 3 & & \\
\hline \multirow[t]{3}{*}{ Procedencia } & No consignado & 0 & 1 & 0 & 1 & 14,908 & ${ }^{*} 0,021$ \\
\hline & Lima & 1 & 18 & 12 & 2 & & \\
\hline & Provincia & 0 & 10 & 17 & 0 & & \\
\hline
\end{tabular}

*significancia estadística: $p<0,05$

Para las características clínicas, se encontró que la vía de ingreso y el tipo (o condición) del paciente es significativa, siendo por Emergencia (x2: 8,331; p: 0,040) y reingresantes (x2: 26,077; p: 0,000) con motivos clínicos (Tabla 4).

Tabla 4. Análisis comparativo de características demográficas con el motivo de ingreso en pacientes con internamiento prolongado

\begin{tabular}{|c|c|c|c|c|c|c|c|}
\hline \multicolumn{8}{|c|}{ Motivo de Ingreso } \\
\hline & & $\begin{array}{l}\text { No } \\
\text { Consignado }\end{array}$ & Clínico & Judicial & $\begin{array}{l}\text { Policial, } \\
\text { Fiscal }\end{array}$ & $\mathbf{X} 2$ & $\mathbf{P}$ \\
\hline \multirow[t]{2}{*}{ Vía de ingreso } & Consulta externa & 1 & 4 & 11 & 0 & 8,331 & ${ }^{*} 0,040$ \\
\hline & Emergencia & 0 & 25 & 18 & 3 & & \\
\hline \multirow[t]{2}{*}{ Tipo de paciente } & Nuevo & 0 & 5 & 23 & 0 & 26,077 & ${ }^{*} 0,000$ \\
\hline & Reingreso & 1 & 24 & 6 & 3 & & \\
\hline \multirow[t]{9}{*}{ Diagnóstico } & No consignado & 0 & 0 & 0 & 0 & 13,650 & 0,324 \\
\hline & Esquizofrenia & 1 & 22 & 13 & 3 & & \\
\hline & Trastorno bipolar & 0 & 1 & 0 & 0 & & \\
\hline & Trastorno depresivo & 0 & 0 & 0 & 0 & & \\
\hline & Trastorno de ansiedad & 0 & 0 & 0 & 0 & & \\
\hline & Trastorno de personalidad & 0 & 2 & 10 & 0 & & \\
\hline & $\begin{array}{l}\text { Trastorno de consumo de } \\
\text { sustancias psicoactivas }\end{array}$ & 0 & 0 & 2 & 0 & & \\
\hline & $\begin{array}{l}\text { Trastorno mental orgánico } \\
\text { y/o retraso mental }\end{array}$ & 0 & 4 & 4 & 0 & & \\
\hline & Otros & 0 & 0 & 0 & 0 & & \\
\hline
\end{tabular}




\begin{tabular}{|c|c|c|c|c|c|c|c|}
\hline \multicolumn{8}{|c|}{ Motivo de Ingreso } \\
\hline & & $\begin{array}{l}\text { No } \\
\text { Consignado }\end{array}$ & Clínico & Judicial & $\begin{array}{l}\text { Policial, } \\
\text { Fiscal }\end{array}$ & $\mathbf{X} 2$ & $\mathbf{P}$ \\
\hline \multirow{3}{*}{$\begin{array}{l}\text { Tipo de } \\
\text { diagnóstico }\end{array}$} & No consignado & 0 & 1 & 0 & 0 & 4,287 & 0,638 \\
\hline & Único & 0 & 19 & 18 & 1 & & \\
\hline & Comórbido & 1 & 9 & 11 & 2 & & \\
\hline \multirow{3}{*}{$\begin{array}{l}\text { Signos y } \\
\text { síntomas (al } \\
\text { ingreso) }\end{array}$} & No consignado & 0 & 0 & 0 & 0 & 7,172 & 0,067 \\
\hline & Con agresividad & 0 & 22 & 15 & 3 & & \\
\hline & Sin agresividad & 1 & 7 & 14 & 0 & & \\
\hline \multirow{4}{*}{$\begin{array}{l}\text { Evolución (en } \\
\text { hospitalización) }\end{array}$} & No consignado & 0 & 0 & 0 & 0 & 1,895 & 0,929 \\
\hline & Favorable & 0 & 11 & 10 & 1 & & \\
\hline & Desfavorable & 0 & 1 & 0 & 0 & & \\
\hline & Estacionaria & 1 & 17 & 19 & 2 & & \\
\hline \multirow[t]{9}{*}{ Tratamiento } & No consignado & 0 & 0 & 0 & 0 & 11,592 & 0,710 \\
\hline & A. típicos & 0 & 2 & 0 & 0 & & \\
\hline & A. atípicos & 0 & 3 & 2 & 0 & & \\
\hline & Clozapina & 0 & 3 & 2 & 0 & & \\
\hline & Antidepresivos & 0 & 0 & 0 & 0 & & \\
\hline & Ansiolíticos & 0 & 0 & 2 & 1 & & \\
\hline & Estabilizadores del ánimo & 0 & 0 & 0 & 0 & & \\
\hline & Combinación de fármacos & 1 & 20 & 23 & 2 & & \\
\hline & Otros & 1 & 1 & 0 & 0 & & \\
\hline
\end{tabular}

*significancia estadística: $p<0,05$

Para las características sociales, hay mayor significancia en aquellos pacientes acompañados por familiares ( $\times 2$ : 14,235; p: 0,027$)$ y quienes ingresan con otras personas distintas a la familia no emparentadas, considerado como "Otros" ( 22 : 22,049; p: 0,037), por motivo clínico (Tabla 5).

Tabla 5. Análisis de características sociales con el motivo de ingreso en pacientes con internamiento prolongado

\begin{tabular}{|c|c|c|c|c|c|c|c|}
\hline \multicolumn{8}{|c|}{ Motivo de Ingreso } \\
\hline & & $\begin{array}{l}\text { No } \\
\text { Consignado }\end{array}$ & Clínico & Judicial & $\begin{array}{l}\text { Policial, } \\
\text { Fiscal }\end{array}$ & $\mathbf{X} 2$ & $\mathbf{P}$ \\
\hline \multirow{3}{*}{$\begin{array}{l}\text { Condición } \\
\text { familiar actual }\end{array}$} & No consignado & 0 & 1 & 1 & 0 & 5,326 & 0,503 \\
\hline & Con abandono sociofamiliar & 0 & 14 & 11 & 3 & & \\
\hline & Sin abandono sociofamiliar & 1 & 14 & 17 & 0 & & \\
\hline \multirow{3}{*}{$\begin{array}{l}\text { Persona que lo } \\
\text { acompaña al } \\
\text { ingreso }\end{array}$} & No consignado & 0 & 3 & 4 & 0 & 14,235 & ${ }^{*} 0,027$ \\
\hline & Con familia & 1 & 23 & 17 & 0 & & \\
\hline & Sin familia & 0 & 3 & 8 & 3 & & \\
\hline \multirow{5}{*}{$\begin{array}{l}\text { Acompañante } \\
\text { al ingreso sin } \\
\text { familia }\end{array}$} & No consigna & 0 & 2 & 2 & 0 & 22,049 & ${ }^{*} 0,037$ \\
\hline & Policía & 0 & 1 & 6 & 3 & & \\
\hline & Amigo & 0 & 0 & 1 & 0 & & \\
\hline & Personal de salud & 0 & 1 & 0 & 0 & & \\
\hline & Otros & 1 & 25 & 20 & 0 & & \\
\hline
\end{tabular}


Características personales, clínicas y sociales en pacientes con estancia hospitalaria prolongada en los pabellones de pacientes agudos del Hospital Víctor Larco Herrera

\begin{tabular}{|c|c|c|c|c|c|c|c|}
\hline \multicolumn{8}{|c|}{ Motivo de Ingreso } \\
\hline & & $\begin{array}{l}\text { No } \\
\text { Consignado }\end{array}$ & Clínico & Judicial & $\begin{array}{l}\text { Policial, } \\
\text { Fiscal }\end{array}$ & X2 & $\mathbf{P}$ \\
\hline \multirow{3}{*}{$\begin{array}{l}\text { Tipo de familia } \\
\text { del paciente }\end{array}$} & No consigna & 0 & 4 & 6 & 2 & 5,495 & 0,482 \\
\hline & Funcional & 0 & 3 & 2 & 0 & & \\
\hline & Disfuncional & 1 & 22 & 21 & 1 & & \\
\hline \multirow{6}{*}{$\begin{array}{l}\text { Soporte familiar } \\
\text { principal }\end{array}$} & No consignado & 0 & 5 & 3 & 0 & 16,137 & 0,373 \\
\hline & Pareja & 0 & 2 & 0 & 1 & & \\
\hline & Padres & 1 & 11 & 19 & 1 & & \\
\hline & Hermanos & 0 & 7 & 6 & 0 & & \\
\hline & Hijos & 0 & 3 & 1 & 1 & & \\
\hline & Otros & 0 & 1 & 0 & 0 & & \\
\hline \multirow{3}{*}{$\begin{array}{l}\text { Comorbilidad } \\
\text { familiar }\end{array}$} & No consignado & 0 & 5 & 5 & 2 & 10,461 & 0,107 \\
\hline & Sí & 0 & 14 & 7 & 1 & & \\
\hline & No & 1 & 10 & 17 & 0 & & \\
\hline \multirow{6}{*}{$\begin{array}{l}\text { Nivel } \\
\text { Socioeconómico }\end{array}$} & No consignado & 0 & 0 & 1 & 0 & 9,239 & 0,415 \\
\hline & $A$ & 0 & 0 & 0 & 0 & & \\
\hline & B & 0 & 0 & 0 & 0 & & \\
\hline & C & 0 & 0 & 1 & 0 & & \\
\hline & $D$ & 0 & 2 & 0 & 1 & & \\
\hline & SIS & 1 & 27 & 27 & 2 & & \\
\hline
\end{tabular}

*significancia estadística: $p<0,05$

Para las características sociales, hay mayor significancia en aquellos pacientes acompañados por familiares ( $\times 2$ : 14,235; p: 0,027$)$ y quienes ingresan con otras personas distintas a la familia no emparentadas, considerado como "Otros" ( $\times 2$ : 22,049; p: 0,037), por motivo clínico (Tabla 5).

\section{DISCUSIÓN}

Los grupos de pacientes más afectados de acuerdo a la edad son los jóvenes (29\%) y jóvenes adultos (32\%), lo cual podría ser debido al alto porcentaje de pacientes judiciales.

La mayoría de pacientes con larga estancia hospitalaria, presentan disfuncionalidad y abandono familiar, apoyándose los resultados con el estudio de Afilalo et al. ${ }^{8}$ quienes obtuvieron un $11 \%$ de pacientes sin hogar antes de ingresar al hospital psiquiátrico y pasar a estancia prolongada.

El 62,9\% de los pacientes con estancia prolongada tienen un diagnóstico definitivo de esquizofrenia, concordando con los estudios de Adegunloye et al. ${ }^{(9)}$, con el mismo diagnóstico en un $55 \%$, y de Afilalo et al. ${ }^{(8)}$ que otorga mayor frecuencia clínica a aquellos cuadros donde predominaban alucinaciones y delirios en un $34 \%$, sin especificar diagnóstico. El segundo diagnóstico que aparece con frecuencia en nuestro estudio son los trastornos de personalidad, superando por mucho a los trastornos afectivos, el cual prevalece en otros trabajos como segundo lugar, no siendo compatibles con otras investigaciones. El 35,5\% de los pacientes, tienen una evolución favorable en hospitalización, pero permanecen internados.

Esto podría ser debido a otros factores sociales y externos, ajenos a la función asistencial. La mayoría también fueron reingresos, siendo esta variable significativa, planteándonos diversas situaciones probables y una de ellas es que, a pesar de la evolución favorable, estos pacientes salen de alta médica y no se les realiza el seguimiento ni control adecuado en los niveles correspondientes y por lo tanto retornan. El motivo de ingreso predominante fue sentencia judicial/policial, mientras que el segundo fueron los factores clínicos. Entre los diagnósticos clínicos están la esquizofrenia, trastorno de personalidad y trastornos orgánicos. Los pacientes judicializados afectan toda la estadística hospitalaria, porque no ajustan su permanencia a una evolución clínica favorable o condición social, sino a 
lo legal, rompiendo con todos los parámetros de medición y estándares establecidos. Estudios como los de Zhang ${ }^{(10)}$, Herr ${ }^{(11)}$ y Addisu ${ }^{(12)}$ apoyan las evidencias en relación a que la esquizofrenia y el criterio clínico predominan en la estancia, no ajustándose al criterio judicial que se encontró en nuestra institución, siendo además, no significativo el diagnóstico en los pacientes con mayor estancia.

Más de la mitad de pacientes procedía de Lima, resultando una variable significativa. Los restantes, procedentes de provincia, se les agrega otros factores sociales como abandono de familia, situación socioeconómica $C \circ D$, con altos porcentajes de presentación en los pacientes estudiados, por lo que las probabilidades de estancia prolongada, es muy alta, tal como Zhang et al. también plantearon; sin embargo, en nuestros hallazgos no resultaron significativos.

Es importante destacar que dos terceras partes de la muestra estuvo recibiendo combinación de fármacos por vía oral (antipsicóticos típicos y atípicos, ansiolíticos, entre otros), sin embargo, en el trabajo de Jiménez et al. ${ }^{(13)}$ a pesar de tomar como factor predictor el uso de psicotrópicos prevalece el de antipsicóticos solos en un $38,5 \%$ asumiendo que no da lugar a combinaciones posteriores, lo cual resulta incomparable a nuestros hallazgos. Nuestro estudio en función a la evolución favorable de los pacientes, deja una vez más abierta la posibilidad de que el factor más importante asociado a la estancia hospitalaria, no es el factor clínico, sino que, a pesar del buen manejo de los pacientes y su evolución favorable, se atribuyen factores sociales, personales y judiciales, principalmente, que escapan al control del equipo de salud del personal y por ende al cumplimiento de los estándares establecidos para una adecuada estancia hospitalaria, concordando también con los hallazgos de Cheng ${ }^{(14)}$, pero contrapuesto a Anderson ${ }^{(15)}$ y Øiesvold ${ }^{(16)}$, que dan mayor importancia a la complejidad clínica (por ejemplo, estados psicóticos y admisiones planificadas) y no otros factores. Otros estudios de predicción de factores clínicos y el tiempo de hospitalización, no llegan a ser compatibles con los hallazgos particulares de nuestro trabajo ${ }^{(17,18,19)}$.

La principal limitación del estudio corresponde a su naturaleza de ser retrospectivo y transversal por la dificultad de obtener información fiable, sobre todo por la búsqueda de factores asociados. Otra limitación fue solamente considerar la muestra de pacientes con estancia mayor de 60 días, por lo que se dificulta evidenciar características generalizadas comparativas con pacientes que tienen menos tiempo en el hospital y mayor confiabilidad de la significancia. Además, el gran número de pacientes judicializados en la muestra limita el criterio clínico, el cual, a pesar de ser un factor de sesgo, nos da una visión de la problemática en esta institución.

En conclusión, el ser de sexo masculino, soltero, proceder de Lima, ingresar por la vía de Emergencia, en condición de reingreso, el estar acompañado por familiares y por otras personas no emparentadas cuando no son familiares, influye en la hospitalización prolongada según el motivo de ingreso sea clínico o judicial. En el Hospital Víctor Larco Herrera el tiempo de estancia hospitalaria se encuentra más afectado probablemente debido al gran número de pacientes que ingresan por vía judicial.

\section{AGRADECIMIENTOS}

Agradecemos a la Dra. Gisella Vargas Cajahuanca, Dr. Luis Vilchez Salcedo y a la Dra. Ana Boza Huamaní por habernos brindado las facilidades para la realización de este estudio.

\section{REFERENCIAS BIBLIOGRÁFICAS}

1. Hopko DR, Lachar D, Bailley SE, Varner RV. Assessing predictive factors for extended hospitalization at acute psychiatric admission. Psychiatr Serv. 2001;52(10):1367-73.

2. Chung W. Psychiatric inpatient expenditures and public health insurance programmes: analysis of a national database covering the entire South Korean population. BMC Health Serv Res. 2010; 10(1):263.

3. Fulop G, Strain JJ, Fahs MC, Hammer JS, Lyons JS. Medical disorders associated with psychiatric comorbidity and prolonged hospital stay. Psychiatr Serv. 1989;40(1):80-2.

4. Defensoría del Pueblo - Ministerio de Salud, Salud Mental y Derechos Humanos: La situación de los derechos de las personas internadas en establecimientos de salud mental. Serie Informes Defensoriales. Informe Defensorial $N^{\circ} 102$. 2005. Disponible en: http://www.minsa.gob.pe/dgsp/ archivo/salud_mental_documentos/15_informe_102_ defensoria_peru_\%20junio.pdf

5. Defensoría del Pueblo - Ministerio de Salud. Salud Mental y Derechos Humanos: Supervisión de la política pública, la calidad de los servicios y la atención a poblaciones vulnerables. Serie Informes Defensoriales. Informe Defensorial N¹40. 2009. Disponible en: http://www.minsa.gob.pe/dgsp/archivo/ salud_mental_documentos/16_informe_defensorial_140.pdf

6. Lyketsos CG, Dunn G, Kaminsky MJ, Breakey WR. Medical comorbidity in psychiatric inpatients: relation to clinical outcomes and hospital length of stay. Psychosomatics. 2002;43(1):24-30.

7. Douzenis A, Seretis D, Nika S, Nikolaidou P, Papadopoulou A, Rizos EN, et al. Factor affecting hospital stay in psychiatric patients: The role of active comorbility. BMC Health Serv Res. 2012; 12(1):166.

8. Afilalo M, Soucy N, Xue X, Colacone A, Jourdenais E, Boivin $J$. Characteristics and needs of psychiatric patients with prolonged hospital stay. Can J Psychiatry. 2015; 60(4):181-8.

9. Adegunloye O, Yussuf A, Ajiboye P, Issa B, Buhari I. Correlates of Length of Stay among Psychiatric In-Patients in a Tertiary Health Institution in Nigeria. Res J of Med Sci. 2009; 3(2): 5661. 
10. Zhang J, Harvey C, Andrew C. Factors associated with length of stay and the risk of readmission in an acute psychiatric inpatient facility: A Retrospective Study. Aus N Zea J Psychiatry. 2011; 45(7):578-585.

11. Herr B, Abraham H, Anderson W. Length of stay in a General Hospital Psychiatric Unit. Gen Hosp Psychiatry. 1991; 13(1): 68-70.

12. Addisu F, Wondafrash $M$, Chemali Z, Dejene T, Tesfaye $M$. Length of stay of psychiatric admissions in a general hospital in Ethiopia. Int J of Ment Health Syst. 2015; 9(1):13.

13. Jiménez RE, Lam RM, Marot M, Delgado A. Observed-predicted length of stay for an acute psychiatric department, as an indicator of inpatient care inefficiencies. Retrospective caseseries study. BMC Health Serv Res. 2004; 4(1):4.

14. Cheng JE, Shumway M, Leary M, Mangurian C. Patient factors associated with extended length of stay in the psychiatric inpatient units of a large urban county hospital. Community Ment Health J. 2016; 52(6): 658-61.

15. Anderson S, Crist A, Payne N. Predicting inpatient length of stay with the expanded version of the Brief Psychiatric Rating Scale (version 4.0). Psychiatr Serv. 2004; 55(1): 77-9.

16. Saarento O, Sytema S, Christiansen L, Göstas G, Lönnerberg $O$, Muus S, et al. The Nordic Comparative Study on Sectorized Psychiatry-length of in-patient stay. Acta Psychiatr Scand. 1999;100(3):220-8.

17. Matsumoto T, Kobayashi T, Kato S. Predictors of length of hospital stay for patients with late-onset depression in Japan. Psychogeriatrics. 2014; 14(2):124-31.

18. Rhodes SM, Patanwala AE, Cremer JK, Marshburn ES, Herman $M$, Shirazi FM, et al. Predictors of Prolonged Length of Stay and Adverse Events among Older Adults with Behavioral Health- Related Emergency Department Visits: A Systematic Medical Record Review. J Emerg Med. 2016;50(1):143-52.
19. Warren MB, Campbell RL, Nestler DM, Pasupathy KS, Lohse $\mathrm{CM}$, Koch KA, et al. Prolonged length of stay in ED psychiatric patients: a multivariable predictive model. The Am J Emerg Med. 2016;34(2):133-9.

Fuentes de financiamiento:

El estudio ha sido financiado por el Hospital Víctor Larco Herrera.

Conflictos de interés:

Los autores declaran no tener ningún conflicto de interés.

Correspondencia:

Roger Abanto Marín

Dirección: Jr. Daniel Hernández 1360, Pueblo Libre

Teléfono: 975528813

Correo electrónico: roabama@gmail.com

Recibido: 24 de noviembre de 2017

Evaluado: 16 de enero de 2018

Aprobado: 13 de febrero de 2018

(C) La revista. Publicado por Universidad de San Martín de Porres, Perú. (сc) ву Licencia de Creative Commons Artículo en acceso abierto bajo términos de Licencia Creative Commons Atribución 4.0 Internacional. (http: //creativecommons.org/licenses/by/4.0/)

ORCID iDs

Roger Abanto

Glauco Valdivieso-Jiménez

Adrián Arévalo

Sandra Orihuela

https: / / orcid.org/0000-0003-4828-6290 https://orcid.org/0000-0001-8561-676 https://orcid.org/0000-0001-6106-396X https://orcid.org/0000-0001-8691-293X 